\title{
Body Shape versus Body Composition as Predictor on Ironman Race Performance
}

\author{
Drs. Michel Kandel \\ Master of Science Physical Therapy, Master in Manual Therapy, Master in musculoskeletal \\ Ultrasound i.e. Institution of Physiotherapy, Oberriet, Switzerland
}

\begin{abstract}
:
Objectives: The aim of this study was to analyze the impact of body shape and body composition on Ironman performance. It was also aimed to give Ironman trainers and coaches a useful and reliable tool to evaluate performance level and physical improvements in the training process.
\end{abstract}

Methods: A total of 119 male and 17 female competitors of the Ironman Switzerland volunteered in this study. Ten anthropometric dimensions were measured and a Bioelectrical Impedance Analysis (BIA) was done for all subjects. The obtained variables were compared with race performance.

Results: The somatotype (body shape) was a strong predictor of Ironman performance $(R=.535 ; p<0.01)$ in male athletes. An ideal somatotype of 1.7 - 4.7 - 3.1 could be established. Body composition hat only a small impact on race performance $(R=.255 ; p<0.01)$. A combination of variables in body shape and body composition can explain for $30.7 \%$ the variance in Ironman performance in male athletes. In female athletes no correlations between body shape, body composition and race performance were found. Body height was the only variable correlating with race time $(r=-.590 ; p<0.05)$.

Conclusions: Body shape is a better predictor than body composition in male Ironman performance. By measuring five anthropometric variables, $28.7 \%$ of the variance in race performance can be predicted. These measurements as well as the ideal somatotype can be used by trainers and coaches to control the training process and the physical improvements of their male Ironman athletes.

Keywords: BIA, Body Fat Mass, Somatotype, Triathlon, Athletes.

\section{INTRODUCTION}

In sports with a major physical component, body shape and body composition play an important role [1] [2]. There is substantial evidence that body shape and body composition are related to sport performance ${ }^{[3]}[4]{ }^{[5]}{ }^{[6]}$. Trainers and coaches therefore use these data as a tool to assess the performance level or improvements of their athletes. However, body shape and body composition have a different impact on different sports, which makes a general practical approach almost impossible. Information about the specific sport and the impact of the factors may contribute to a more reliable statement.

An Ironman is a long distance triathlon with duration between eight and seventeen hours ${ }^{[7]}$. Athletes who perform in such an event need to prepare for long distance competition in three disciplines (swim, bike, run). This indicates a high level of physical activity over several years with adaptations in body shape and body composition.

The aim of this study was to analyze the impact of body shape and body composition on the performance in an Ironman race. It was also aimed to find out, which of these variables are most useful in practice and may operate as a reliable assessment for trainers and coaches in guiding Ironman athletes.

\section{MATerial AND MeTHOD}

Hundred twenty-two male and 18 female athletes out of 1'674 competitors of the Ironman Switzerland volunteered in this study. In all subjects' body composition and body shape was estimated. Body shape was quantified by the somatotype method, according to Heath \& Carter 


\section{Drs. Michel Kandel}

Protocol ${ }^{[8]}$ and the Body Mass Index (BMI). Therefore 10 anthropometric dimensions were taken. The measurements were made within three days prior to the event by three trained anthropometric investigators (Intraclass Correlation Coefficient, ICC, was $r=1.00$ for all) and performed according to the International Society for the Advancement of Kinanthropometry (ISAK) Manual ${ }^{[9]}$. Besides body height (Stadiometer: GPM, Seritex, Inc., Carlstadt, New Jersey, model 101) and weight (Tanita Corporation, Tokyo, Japan, Model BC-532), four skinfolds with a Slimguide Skinfold Caliper (triceps, subscapular, supraspinal, medial calf), two bone breadths with a Campbell Small Bone Caliper, Rosscraft, Canada, model CAM 10 (biepicondylar humerus and femur) and two limb girths with a flexible metallic tape measure (Rosscraft, Canada) (arm flexed and tensed, calf) were taken. All anthropometric dimensions - except stature and weight - were taken twice. For the calculation, the duplicated measurements were averaged and rounded to the nearest $0.5 \mathrm{~mm}$ (skinfolds and bone breaths), resp. $0.1 \mathrm{~mm}$ (limb girths). The calculation of the somatotype of each individual was made by the equations published by Heath and Carter [8]. The calculation of the somatotype gave numbers for each of the somatotype components. The endomorphy component represents the relative adiposity, the mesomorphy component stands for the relative musculoskeletal robustness, and the ectomorphy component represents the relative linearity or slenderness. The BMI was calculated by dividing weight through heigth ${ }^{2}$.

In a field research under controlled conditions a reliable quantification of body composition can be made by a bioelectrical impedance analysis (BIA) ${ }^{[10]}$. A fourth trained investigator measured all subjects under standardized conditions with a Biacorpus RX-4000 (MEDI CAL HealthCare GmbH, Karlsruhe, Germany). They all had to lay supine on a cushioned wooden table for at least 5 to 10 minutes. Arms and legs were separated from the trunk by about 30 to $45^{\circ}$. The skin was cleaned with alcohol before new adhesive electrodes for each measurement were placed on both hands and feet. The investigator made sure the cables of the electrodes did not touch each other. The Biacorpus RX4000 is a phase sensitive four channel device, which measures with a single frequency of $50 \mathrm{kHz}$. The raw data in terms of Resistance (Rz), Reactance (Xc) was obtained. This data was available for different body segments: RARF (right arm right foot), RALA (right arm left arm), RFLF (right foot left foot) and LALF (left arm left foot). As most studies are based on analysis of the right body side, the calculation of body composition in this study the RARF data was used. The fat-free mass (FFM) was calculated with a validated equation described by Kyle et al. ${ }^{[11]}$ for healthy subjects between 20 and 94 years:

FFM $=-4.104+\left(0.518 \times\right.$ height $\left.^{2} / \mathrm{Rz}\right)+(0.231 \mathrm{x}$ weight $)+(0.130 \mathrm{x} \mathrm{Xc})+(4.229 \times$ sex $[$ men $=1 ;$ women $=0]$ ).

The complete official ranking list was provided by the Ironman organisation. The total race time, split performance in the swim, bike and run discipline, as well as the transition time was at disposal. All the volunteering competitors were informed about the testing procedures. According to the declaration of Helsinki, about the ethical principles for medical research involving human subjects, they were getting full protection of privacy and were able to withdraw their participation in this investigation at any time. They confirmed their permission to use their data by signing a statement of agreement. This research was approved by the ethic commission of the Kanton St. Gallen, Switzerland with registration number EKSG 12/049.

All data was subject to descriptive and inferential analyses, which were made with IBM SPSS Statistics 21 for Windows. All data was checked for assumptions. Parametric tests like Pearson product-moment correlations, linear regression analyses or ANOVAs were only made on continuous ("scale") or interval and independent variables, which were normally distributed. The normal distribution was checked visually (P-P Plots) and with skewness and kurtosis (acceptable range was -1 to 1). Correlation analyses were interpreted in effect size with a Pearson $r$. A correlation coefficient of $r \geq .10$ was defined as a small (weak) effect; $r \geq 0.30$ as a medium (moderate) effect and an $r \geq .50$ as a large (strong) effect ${ }^{[12]}$.

\section{Results}

Three male and one female athlete did not finish the race within the time limit of 16 hours and had to be excluded from this study, as they could not be called an Ironman. The sample consisted of 119 
male athletes aged 24 to 60 years (mean $40.3 \pm 8.1)$ and 17 female athletes ages 29 to 55 years $(40.2 \pm$ 8.3). The results for male and female athletes (Table 1) will be discussed separately.

Table1. Overview of data of the 119 male and 17 female Ironman athletes, with somatotype in units ( $s d=$ standard deviation), Resistance (Rz) and reactance (Xc) in Ohm. Fat mass (FM) and Fat free mass (FFM) in kg and percentage.

\begin{tabular}{|c|c|c|c|}
\hline & & Male & Female \\
\hline & $\mathrm{N}$ & 119 & 17 \\
\hline & Age (years) & 40.3 & 40.2 \\
\hline & $\mathrm{Sd}$ & 8.1 & 8.3 \\
\hline & Height $(\mathrm{cm})$ & 177.7 & 165.1 \\
\hline & $\mathrm{Sd}$ & 6.9 & 4.8 \\
\hline & Weight $(\mathrm{kg})$ & 78.5 & 58.8 \\
\hline & $\mathrm{Sd}$ & 7.7 & 6.2 \\
\hline \multirow{8}{*}{ 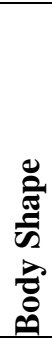 } & Endomorphy & 2.4 & 2.5 \\
\hline & $\mathrm{Sd}$ & 0.7 & 1.0 \\
\hline & Mesomorphy & 5.3 & 4.2 \\
\hline & $\mathrm{Sd}$ & $\mathbf{1 . 0}$ & 1.0 \\
\hline & Ectomorphy & 2.3 & 2.7 \\
\hline & $\mathrm{Sd}$ & 1.0 & 1.0 \\
\hline & BMI $\left(\mathrm{kg} / \mathrm{m}^{2}\right)$ & 24.0 & 21.6 \\
\hline & $\mathrm{sd}$ & 2.1 & 2.3 \\
\hline \multirow{12}{*}{ 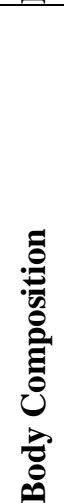 } & Resistance (Rz) & 458.5 & 558.0 \\
\hline & $\mathrm{sd}$ & 45.8 & 45.3 \\
\hline & Reactance (Xc) & 56.5 & 64.6 \\
\hline & $\mathrm{sd}$ & 6.0 & 5.3 \\
\hline & $\mathrm{FM}(\mathrm{kg})$ & 14.8 & 15.4 \\
\hline & sd & 3.9 & 4.0 \\
\hline & FFM $(\mathrm{kg})$ & 61.0 & 43.3 \\
\hline & $\mathrm{sd}$ & 5.2 & 2.8 \\
\hline & FM $(\%)$ & 19.3 & 25.9 \\
\hline & $\mathrm{sd}$ & 3.7 & 4.2 \\
\hline & FFM $(\%)$ & 80.7 & 74.1 \\
\hline & sd & 3.7 & 4.2 \\
\hline \multirow{10}{*}{ 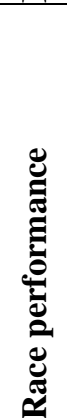 } & Swim (min) & 76.8 & 78.8 \\
\hline & sd & 9.8 & 10.9 \\
\hline & Bike (min) & 357.4 & 397.4 \\
\hline & $\mathrm{sd}$ & 36.7 & 50.9 \\
\hline & Run (min) & 254.4 & 267.8 \\
\hline & $\mathrm{sd}$ & 43.6 & 36.2 \\
\hline & Transitions (min) & 8.6 & 8.7 \\
\hline & $\mathrm{sd}$ & 4.1 & 3.6 \\
\hline & Total Race Time (min) & 697.2 & 752.6 \\
\hline & sd & 83.9 & 91.4 \\
\hline
\end{tabular}

The mean somatotype of all male athletes was $2.4( \pm 0.7)$ for the endomorphy value, $5.3( \pm 1.0)$ for the mesomorphy value, and $2.3( \pm 1.0)$ for the ectomorphy value. The mean weight and height was $75.8 \mathrm{~kg}( \pm 7.7)$ and $177.7 \mathrm{~cm}( \pm 6.9)$, which resulted in a mean BMI of $24.0 \mathrm{~kg} / \mathrm{m}^{2}( \pm 2.1)$. The mean Ironman race time was 697.2 minutes $( \pm 83.9)$ or 11:37:20 hours.

With a linear multiple regression of the somatotype components endomorphy, mesomorphy and ectomorphy on race performance, a significant model $(\mathrm{p}<0.01)$ was found. With a $\mathrm{R}=.535$, and a $\mathrm{R}^{2}$ $=.287$ the somatotype explains for $28.7 \%$ the variance in Ironman performance. However, not all the components contribute equally to the model. The mesomorphy value did not have a significant contribution to the regression model. By eliminating the mesomorphy value of the model the results of the regression model did not change (Table 2). The endomorphy value had a $\beta$ - value of .346 ( $p<$ 0.01 ), which means that a decrease of endomorphy by one standard deviation (and constant ectomorphy value) leads to a reduction in race time of 29 minutes. An increased ectomorphy value by one standard deviation (and constant endomorphy value) leads to an improved race performance of 22 minutes $(\mathrm{p}<0.01)$. 
Table2. Measurement of endomorphy and ectomorphy with 5 anthropometric measurements can predict Ironman race performance for $28.7 \%$.

\begin{tabular}{|c|c|c|c|c|}
\hline \multicolumn{5}{|c|}{ MEASUREMENT 1} \\
\hline Description & Measurements & $\begin{array}{l}\text { Impact of } 1 \text { standard } \\
\text { deviation }\end{array}$ & Impact of 1 unit & $\begin{array}{l}\text { Total impact on } \\
\text { race performance }\end{array}$ \\
\hline $\begin{array}{l}\text { Endomorphy } \\
\text { value } \\
\text { (unit) }\end{array}$ & $\begin{array}{l}3 \text { skinfolds } \\
\text { Height }\end{array}$ & 29.0 minutes & 39.5 minutes & \\
\hline $\begin{array}{l}\text { Ectomorphy } \\
\text { value } \\
\text { (unit) }\end{array}$ & $\begin{array}{l}\text { Weight } \\
\text { Height }\end{array}$ & - 22.0 minutes & - 22.5 minutes & \\
\hline Total & 5 & & & $28.7 \%$ \\
\hline
\end{tabular}

The Body Mass Index (BMI) had also a significant $(\mathrm{p}<0.01)$ correlation with race performance. With a $\mathrm{R}=.453$ and $\mathrm{R}^{2}=.205$, the variance in race performance depends for $20.5 \%$ on the BMI. Changes in BMI by one standard deviation lead to changes in race performance of 38 minutes.

All somatotype components, as well as the BMI, had the greatest impact on the run discipline and the least impact on the swim discipline. The endomorphy component had an impact of $24.8 \%$ on the run performance $(\mathrm{p}<0.01)$.

The BIA gave results in resistance and reactance. FFM and FM in kilograms as well as FFM and FM in percent could be calculated and were subject to descriptive and multiple linear regression analyses. FFM in all male athletes was $61.0 \mathrm{~kg}( \pm 5.2)$ or $80.7 \%( \pm 3.7)$, the FM was $14.8 \mathrm{~kg}( \pm 3.9)$ or $19.3 \%$ $( \pm 3.7)$. The regression model of FM and FFM $(\mathrm{R}=.255 ; \mathrm{p}<0.01)$ in kilograms was slightly superior to the regression model of FM and FFM in percent $(\mathrm{R}=.236 ; \mathrm{p}=0.01)$. The variance in race performance however, depended only for $6.5 \%$ on the body composition. Within the regression analysis the FFM did not contribute significantly. Decrease in FM by one standard deviation leads to an improved race performance of 21.4 minutes. The FM had also the biggest impact on the run performance $(\mathrm{R}=.249 ; \mathrm{p}<0.01)$.

The mean somatotype of all female athletes was $2.5( \pm 1.0)$ for the endomorphy value, $4.2( \pm 1.0)$ for the mesomorphy value and $2.7( \pm 1.0)$ for the ectomorphy value. The mean weight and height was $58.8 \mathrm{~kg}( \pm 6.2)$ and $165.1 \mathrm{~cm}( \pm 4.8)$, which resulted in a mean BMI of $21.6 \mathrm{~kg} / \mathrm{m}^{2}( \pm 2.3)$. The mean Ironman race time was 752.6 minutes $( \pm 91.4)$ or 12:12:36 hours. The mean body composition in terms of FFM and FM was $43.3 \mathrm{~kg}( \pm 2.8)$ or $74.1 \%( \pm 4.2)$ and $15.4 \mathrm{~kg}( \pm 4.9)$ or $25.9 \%( \pm 4.2)$ respectively. Body height was the only variable that had a significant correlation with the total race time $(r=-.590 ; \mathrm{p}<0.05)$. Taller female athletes performed significantly better irrespective of their body shape and body composition.

\section{DISCUSSION}

In male Ironman athletes, variables as age, height, weight, BMI, somatotype, FM, as well as FFM had all significant correlations with Ironman race performance. These findings are supported by literature. In 1997 Landers et al. ${ }^{[13]}$ Measured 71 triathletes (49 male, 38 female) competing in the world championship in olympic distance and came to the result that the total race time and run time were slower with an increased level of adiposity. In 2010 Knechtle ${ }^{[14]}$ found in 27 male and 16 female Ironman athletes, a significant positive relationship between percentage body fat and total race time in male, but not in female athletes. In 2009 and 2010 Knechtle ${ }^{[15]}$ studied 184 recreational male Ironman athletes and found that a lower body mass and lower body fat were associated with a faster Ironman race and a faster run split.

As the impact of the different variables as well as their interaction had not yet been studied, it was not clear, which of the variables had the greatest impact on performance. It was also unknown how the interaction between the variables worked out. A multiple regression analysis made these interactions clear. It could be determined that body shape in male Ironman athletes explains for $28.7 \%$ the variance in race performance. Body shape was a better predictor than body composition in terms of FM and FFM. The somatotype described the body shape better than the BMI. Age did not contribute significantly to any regression model on race performance. This means that the correlation between age and performance may be due to age related changes in body shape. 
Combining body shape and body composition in a multiple regression model on race performance revealed significant impact factors. The model with a combination of the three significant factors endomorphy, BMI and FM had a $\mathrm{R}^{2}$ of .307 , meaning that this model explains for $30.7 \%$ the variance in race performance (Table 3 ).

Table3. Measurement of endomorphy, BMI and FM by a BIA can predict Ironman race performance for $30.7 \%$.

\begin{tabular}{|l|l|l|l|l|}
\hline MEASUREMENT 2 & Measurements & $\begin{array}{l}\text { Impact of 1 standard } \\
\text { deviation }\end{array}$ & Impact of 1 unit & $\begin{array}{l}\text { Total impact on } \\
\text { race performance }\end{array}$ \\
\hline $\begin{array}{l}\text { Eescription } \\
\text { value } \\
\text { (unit) }\end{array}$ & $\begin{array}{l}3 \text { skinfolds } \\
\text { Height }\end{array}$ & 34.9 minutes & 47.4 minutes & \\
\hline $\begin{array}{l}\text { BMI } \\
\left(\mathbf{k g} / \mathbf{m}^{\mathbf{2}}\right)\end{array}$ & $\begin{array}{l}\text { Weight } \\
\text { Height }\end{array}$ & 29.9 minutes & 14.4 minutes & \\
\hline $\begin{array}{l}\text { FM } \\
(\mathbf{k g})\end{array}$ & BIA & -18.7 minutes & -4.8 minutes & \\
\hline Total & $5+$ BIA & & & $\mathbf{3 0 . 7 \%}$ \\
\hline
\end{tabular}

Interestingly, this model reveals that FM had a negative impact on race time (more fat mass results in better performance), although FM had a positive correlation with race time (more fat correlates with higher race time). The model says that an increased FM by unchanged BMI and endomorphy value leads to a better performance. If FM increases without increased weight (BMI) and without having thicker skinfolds (endomorphy) this FM is not located subcutaneously.

As there was a significant strong relationship between somatotype and race performance, there must also be an ideal somatotype. Out of the regression equations and the best performance time an ideal endomorphy value of 1.7 could be determined. For the mesomorphy this value was 4.7 and for the ectomorphy this value was 3.1. In literature no information about the somatotype of Ironman athletes was found. Travill, et al. ${ }^{[16]}$ reported a mean somatotype of $1.7-4.3-3.1$ in "well-trained" triathletes $(\mathrm{n}=12)$.

In female athletes no correlations between body shape, body composition and race performance was found. Only body height had a significant correlation with race performance. Taller female athletes performed better than smaller athletes. Out of the regression equation an ideal height of 176 centimeter was found. The mean somatotype of these female athletes was 2.5-4.2-2.7. Leak and Carter ${ }^{[17]}$ found a mean somatotype in trained female athletes $(n=16)$ of $3.1-4.3-2.6$. It is not clear, why there was not found any correlation between performance and somatotype, BMI or FM. It might be due to the relatively small number of female athletes $(n=17)$ or due to the fact that Ironman performance in female athletes depends on other variables than body shape and body composition. There are other investigators, who didn't find these correlations either ${ }^{[14]}$.

\section{CONCLUSION}

Body shape is a better predictor than body composition in Ironman race performance. The somatotype of a male Ironman athlete explains for $28.7 \%$ the variance in Ironman race time. A trainer or coach needs to obtain five measurements (height, weight and 3 skinfolds) to have a reliable assessment in evaluating performance level and improvements of their male Ironman athletes. An additional BIA can give a two percent higher prediction to this regression model. The model also gave information about the impact of each variable on the total race time. Thus, a reduction in the endomorphy value by 0.1 units leads to a better race performance of four minutes. Decrease in endomorphy by one standard deviation ( 0.7 units) improves performance by 29 minutes.

The ideal somatotype of $1.7-4.7-3.1$ can be used as a reference frame for Ironman trainers and coaches to control the training process and to improve talent detection and identification of Ironman athletes. The variance in race performance does not depend on age. Changes in body shape, which can be due to age, do have impacts on race performance of male athletes.

In female athletes, no relationship between body shape, body composition and performance was found. Only body height had a significant relationship with race performance. An optimal body height of 1.76 meter could be established in these 17 female athletes. More research in female athletes, especially in bigger samples and with other variables, could give more information about predictors in their Ironman performance. 


\section{Practical Implications}

- Body shape, obtained with five anthropometric measurements (height, weight, 3 skinfolds), canpredict for $28.7 \%$ the Ironman race performance of male athletes.

- Additional analysis of body composition (BIA) can increase this prediction in performance to $30.7 \%$

- For male Ironman athletes an ideal somatotype of $1.7-4.7-3.1$ could be established.

- In female athletes no relationship between body shape, body composition and race performance was found.

\section{ACKNOWLEDGEMENTS}

The author would like to thank the organising committee of the Ironman Switzerland and all the volunteered athletes. There was no financial support involved in this research.

\section{REFERENCES}

[1] J. Carter and B. Heath, Somatotyping; Development and Applications, Cambridge: Cambridge University Press, 2005.

[2] D. Kerr and A. Stewart, "Body Composition in Sport," in Applied Anatomy and Biomechanics in Sport, Human Kinetics, 2009.

[3] B. Knechtle, A. Wirth, C. Rüst and T. Rosemann, "The Relationship between anthropometry and split performance in recreational male Ironman athletes," Asian Journal of Sports Medicine, vol. 2, no. 1, pp. 23-30, March 2011.

[4] G. Slater, A. Rice, I. Mujika, A. Hahn and K. Sharp, "Physique trails of lightweight rowers and their relationship to competitve success," British Journal of Sports Medicine, pp. 736-741, 2005.

[5] D. Ugarkovic, D. Matavulj and M. Kukolj, "Standard Anthropometric, Body Composition, and Strength Variables as Predictors of Jumping Performance in Elite Junior Athletes," Journal of Strength and Conditioning Research, pp. 227-230, 2002

[6] M. Kandel, J. Baeyens and P. Clarys, "Somatotype, Training and Performance in Ironman Athletes," European Journal of Sport Science, vol. 14, no. 4, pp. 301-308, 2014.

[7] B. Babbit, 25 Years of the Ironman Triathlon World Championship, Oxford: Meyer und Meyer, 2003.

[8] J. Carter, "The Heath-Carter anthropometric somatotype; instruction manual," Surrey, Canada, March 2002.

[9] M. Marfell-Jones, K. Norton, J. Carter and T. Olds, International Standards for Anthropometric Assessment, International Society for the Advancement of Kinanthropometry, 2001

[10] U. Kyle, I. Bosaeus, A. De Lorenzo, P. Deurenberg, M. Elia, J. Gómez, B. Heitmann, L. KentSmith, J. Melchior, M. Pirlich, H. Scharfetter, M. Schols and C. Pichard, "Bioelectrical impedance analysis - Part II: utilization in clinical practice," Clinical Nutrition, pp. 1430-1453, 2004.

[11] U. Kyle, L. Genton, L. Karsegard, D. Slosman and C. Pichard, "Single prediction equation for bioelectrical impedance analysis in adults aged 20-94 years.," Nutrition, pp. 248-253, 2001.

[12] Field, Discovering Statisitcs using SPSS, London: SAGE, 2009.

[13] G. Landers, B. Blanksby, T. Ackland and D. Smith, "Morphology and Performance of World Championship triathletes," Annals of human biology, no. 4, pp. 387-400, 2000.

[14] B. Knechtle, A. Wirth, B. Baumann and P. Knechtle, "Differential correlations between anthropometry, training volume, and performance in male and female Ironman triathletes," Journal of Strenth and Conditional Research, no. 24, pp. 2785-93, 2010.

[15] B. Knechtle and e. a. A. Wirth, "The Relationship between anthropometry and split performance in Ironman athletes," Asian recreational male Journal of Sports Medicine, vol. 2, no. 1, pp. 23 30, March 2011. 
[16] Travill and K. D. J.E.L. Carter, "Antropometric characteristics of elite male athletes," in Acces to Active Living: Proceedings of the 10th Commonwealth and International Scientific Congress, Canada, 1994.

[17] J. C. Leake, "Comparison of body composition and somatotype of trained female triathletes," Journal of Sport Sciences, pp. 125-135, 1991

[18] Knechtle, A. Wirth, B. Baumann and P. Knechtle, "Personal best time, percent body fat, and training are differently associated with race time for male and female Ironman athletes," Research Quarterly for Exercise and Sport, no. 1, pp. 62-8, 2010

[19] B. Knechtle, P. Knechtle and T. Rosemann, "Upper body skinfold thickness is related to race performance in male Ironman triathletes," International Journal of Sports Medicine, no. 1, pp. 20-7, 2011.

Citation: M. Kandel."Body Shape versus Body Composition as Predictor on Ironman Race Performance." International Journal of Sports and Physical Education (IJSPE), vol 3, no. 3, 2017, pp. 9-15. doi:http://dx.doi.org/10.20431/2454-6380.0303002.

Copyright: (C) 2017 Authors. This is an open-access article distributed under the terms of the Creative Commons Attribution License, which permits unrestricted use, distribution, and reproduction in any medium, provided the original author and source are credited. 